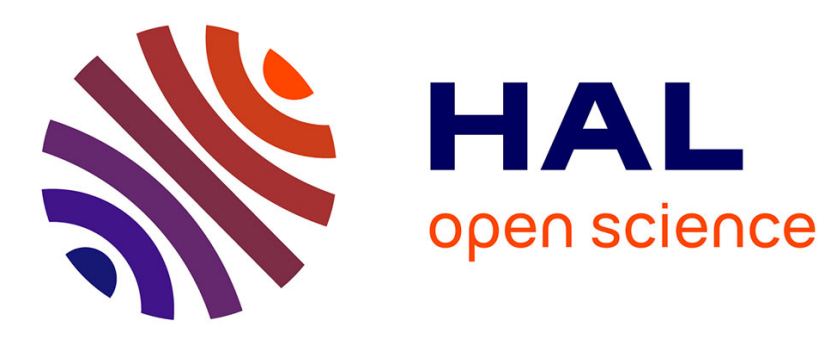

\title{
Minimal perturbations approaching the edge of chaos in a Couette flow
}

Stefania Cherubini, Pietro de Palma

\section{To cite this version:}

Stefania Cherubini, Pietro de Palma. Minimal perturbations approaching the edge of chaos in a Couette flow. Fluid Dynamics Research, 2014, 46 (041403), pp.041403. 10.1088/0169-5983/46/4/041403 . hal-01086726

\section{HAL Id: hal-01086726 \\ https://hal.science/hal-01086726}

Submitted on 24 Nov 2014

HAL is a multi-disciplinary open access archive for the deposit and dissemination of scientific research documents, whether they are published or not. The documents may come from teaching and research institutions in France or abroad, or from public or private research centers.
L'archive ouverte pluridisciplinaire HAL, est destinée au dépôt et à la diffusion de documents scientifiques de niveau recherche, publiés ou non, émanant des établissements d'enseignement et de recherche français ou étrangers, des laboratoires publics ou privés. 


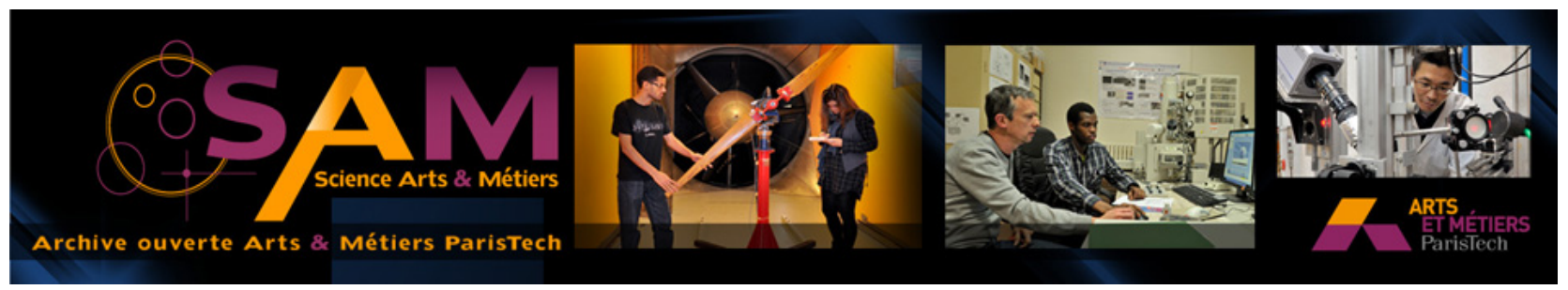

Science Arts \& Métiers (SAM)

is an open access repository that collects the work of Arts et Métiers ParisTech researchers and makes it freely available over the web where possible.

This is an author-deposited version published in: http://sam.ensam.eu Handle ID: .http://hdl.handle.net/10985/8971

\section{To cite this version :}

Stefania CHERUBINI, Pietro DE PALMA - Minimal perturbations approaching the edge of chaos in a Couette flow - Fluid Dynamics Research - Vol. 46, n041403, p.041403 - 2014 


\title{
Minimal perturbations approaching the edge of chaos in a Couette flow
}

\author{
S. Cherubini ${ }^{1}$, P. De Palma ${ }^{2}$
}

${ }^{1}$ DynFluid, Arts et Métiers ParisTech, 151, Bd. de l'Hopital, 75013 Paris, France

E-mail: s.cherubini@gmail.com

${ }^{2}$ DIMMM and CEMeC, Politecnico di Bari, via Re David 200, 70125 Bari, Italy

\begin{abstract}
.
This paper provides an investigation of the structure of the stable manifold of the lower branch steady state for the plane Couette flow. Minimal energy perturbations to the laminar state are computed, which approach within a prescribed tolerance the lower branch steady state in a finite time. For small times, such minimalenergy perturbations maintain at least one of the symmetries characterising the lower branch state. For a sufficiently large time horizon, such symmetries are broken and the minimal-energy perturbations on the stable manifold are formed by localized asymmetrical vortical structures. These minimal-energy perturbations could be employed to develop a control procedure aiming at stabilizing the low-dissipation lower branch state.
\end{abstract}

Keywords: 


\section{Introduction}

A thorough theory of the stability for wall-bounded shear flows is still lacking despite the fact that this kind of flows are ubiquitous in Nature (Hof et al. 2004, Schmid \& Henningson 2001). Scientists have been working on it for 130 years since the seminal work of Reynolds (Reynolds 1883); due to the evident complexity of the matter, they often attack the problem resorting to simple flow configurations such as the plane Couette flow (pCf). For such a flow the laminar solution is linearly stable, so that the onset of a chaotic state cannot be connected to an infinitesimal perturbation of the laminar velocity profile. Instead, transition can be achieved by a sufficiently high-amplitude perturbation for large enough values of the Reynolds number. From a dynamical-system viewpoint, the laminar state is a fixed point in the state space surrounded by its basin of attraction. The boundary of this basin, also called laminarturbulent boundary or edge of chaos (Skufca et al. 2006), is the topic of this paper since its complex structure becomes relevant for understanding the mechanism of transition. In fact, the trajectories starting beyond this boundary evolve towards a disordered state that, in general for the pCf, is a chaotic saddle with a constant probability of decay (Eckhardt et al. 2007). This means that "almost" all trajectories in the state space end up in the fixed point: some of them, starting inside the basin of attraction, relax smoothly; others follow a more or less long disordered route and eventually escape towards the laminar attractor. But there are some trajectories which evolve towards a relative attractor lying on the laminar-turbulent boundary which is called edge state (Skufca et al. 2006).

For the case of the $\mathrm{pCf}$ in a small box, at a Reynolds number $R e_{s n}$, a saddle node bifurcation appears. At the bifurcation point, the upper branch (UB) state is stable and the lower branch (LB), first found by Nagata (1990), Clever \& Busse (1992), has only one unstable direction (Waleffe 2003, Wang et al. 2007). Increasing Re beyond a certain threshold, the UB experiences secondary bifurcations leading to a disordered trajectory in the state space; whereas, the LB maintains a single unstable direction only (Schneider et al. 2008). The stable manifold of the LB state divides the state space into two regions: initial conditions from one side decay smoothly to the laminar profile; initial conditions on the other side flow through a disordered dynamics (see the discussion about turbulence lifetimes in Schneider et al. (2010)). This suggests that the laminarturbulent boundary coincides with this stable manifold and the LB state coincides with the edge state (Schneider et al. 2008). Since the stable manifold of the LB state has an intermediate dissipation rate between turbulent and laminar states, exploring its structure is important for designing a control strategy aiming at avoiding transition to chaos. A control strategy exploiting the dynamics of a codimension-one periodic orbit on the laminar-turbulent boundary has been developed by Kawahara (2005) for inducing relaminarization of a Couette flow. Moreover, perturbations approaching the lower branch solution and evolving along its stable manifold have been investigated by Viswanath \& Cvitanović (2009) for a pipe flow. The authors used a linear combination of 
rolls together with the two unstable modes of the concerned travelling wave (see Pringle \& Kerswell (2007)) for hitting such unstable solution starting from the laminar state. However, such studies haven't investigated the minimal energy threshold characterizing the perturbations on the stable manifold of the concerned unstable solution. An insight on such minimal energy thresholds may be crucial for the establishment of effective control strategies.

Starting from the 90's, the problem of finding a lower bound in terms of perturbation energy/amplitude for reaching transition in shear flows has interested many researchers. The first attempt for determining such threshold amplitudes has been performed by Reddy et al. (1998) which have provided a neutral curve for streak instability using linear stability analysis. Some years later, Cossu (2005) has determined lower bounds for transition using the Waleffe (1997) toy model, whereas Duguet et al. (2010) have computed minimal-energy perturbations inducing transition using a combination of a finite number of linear optimal modes. However, relying on low-dimensional models or on expansions on a basis of modes, such analyses could not accurately describe the minimal seed for turbulence transition, i.e., the perturbation of minimal energy which is able to induce transition in the considered flow. Very recently, a more thorough attempt has been made to search for the perturbations of minimal energy on the laminarturbulent boundary for the pipe (Pringle \& Kerswell 2010, Pringle et al. 2012), the boundary layer (Cherubini et al. 2010, Cherubini et al. 2011) and the pCf (Monokrousos et al. 2011, Rabin et al. 2012, Cherubini \& De Palma 2013, Duguet et al. 2013) flows. To determine the initial condition of minimal energy leading eventually to the edge state (i.e., the minimal seed of turbulent transition), these authors optimize at a large target time a functional linked to the turbulent dynamics (the perturbation kinetic energy (Pringle \& Kerswell 2010, Cherubini et al. 2010, Pringle et al. 2012, Rabin et al. 2012, Cherubini \& De Palma 2013, Cherubini et al. 2011) or the time-averaged dissipation (Monokrousos et al. 2011, Duguet et al. 2013)), and then bisect the initial energy of such "optimal" perturbation until reaching the laminar-turbulent boundary. The outcome of these works shows some similarities among different shear flows: a localized disturbance characterized by large values of the three components of the velocity and composed by staggered vortices has been found in all of the cases. However, some differences have been also recovered. For instance, some of these minimal seeds show a symmetry in the spanwise direction, such as for the case of the boundary layer flow. Concerning the plane Couette flow, some of these minimal perturbations have been found to reach the steady lower branch solution (Duguet et al. 2010, Rabin et al. 2012), whereas some others don't, probably due to the larger Reynolds number considered (Monokrousos et al. 2011, Duguet et al. 2013).

Thus, such a procedure has been shown to be able to find the perturbation of minimal energy which brings the flow in the neighborhood of the edge of chaos in a rather large time and then to turbulence. However, there can be perturbations able to reach the edge in a much shorter time, which may have an initial energy larger but comparable with the "asymptotic" minimum one. Such perturbations may be the key for a shear-flow 


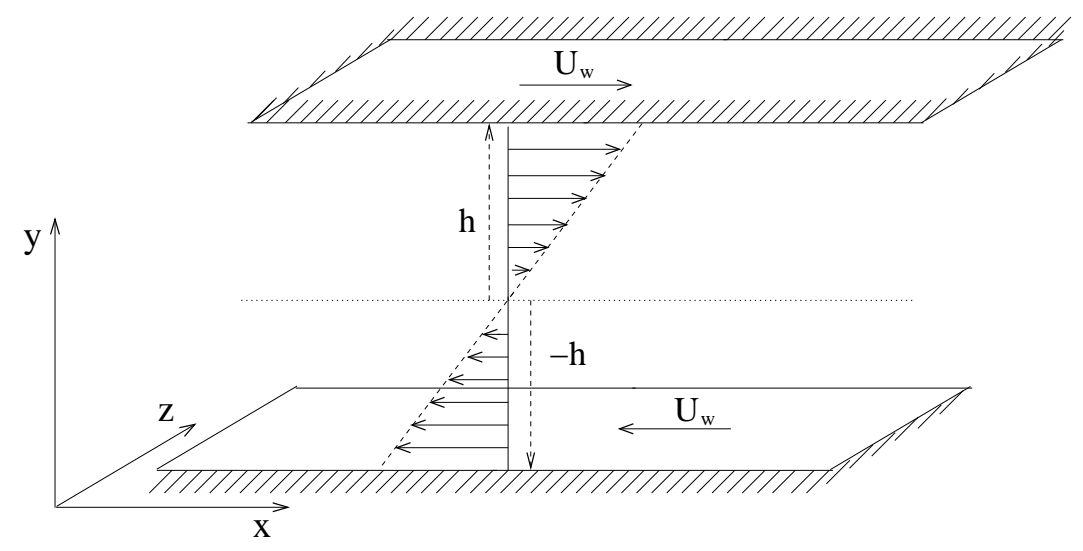

Figure 1. Sketch of the plane Couette flow.

control strategy based on the stabilization of unstable states with low dissipation rate, such as the edge state, exploiting the sensitivity of the system to small disturbances (Pyragas 1992).

The aim of this work is to look for the minimal-energy perturbations to the laminar state lying on the laminar-turbulent boundary using the time as a parameter. Thus, we sample in a systematic way the structure of the laminar-turbulent separatrix, extracting low-energy disturbances to the laminar state from different regions of the phase space. The method used to compute such perturbations is detailed in section 2, whereas the results are given and discussed in section 3. The last section provides a summary of the main results of the work.

\section{Problem formulation}

Let us consider the flow between two parallel plates separated by a distance $2 h$, translating with opposite velocities $\pm U_{w}$, as sketched in Figure 1. Between such plates, a laminar flow in the $x$ direction is established, known as plane Couette flow, having linear profile:

$$
U(y)=U_{w} \frac{y}{h},
$$

where $y$ is the wall-normal direction, whereas the spanwise direction is indicated with $z$. Dimensionless variables are defined with respect to half of the velocity difference between the two walls, $U_{w}$ and half of the distance between the plates, $h$, so that the Reynolds number is $R e=U_{w} h / \nu, \nu$ being the kinematic viscosity. At the walls, the noslip boundary condition is prescribed, whereas in the spanwise and streamwise direction periodicity is imposed for the three velocity components. In the case considered here, a small computational box with $L_{x} \times L_{y} \times L_{z}=4 \pi \times 2 \times 2 \pi$ has been considered, which is the same domain used in Schneider et al. (2008) for the computation of the edge state, and in Monokrousos et al. (2011) for the computation of the minimal perturbation reaching the edge of turbulence. In this domain, the Navier-Stokes (NS) equations 
are discretized on a $201 \times 100 \times 61$ points grid by a finite-difference fractional-step method with second-order accuracy in space and time (Verzicco \& Orlandi 1996). The code has been validated by computing, using an edge tracking procedure, the steady lower branch solution of Schneider et al. (2008), at $R e=400$. The typical solution is characterized by a bent streaky structure flanked by streamwise vortices, characterized by two symmetries: a finite translation symmetry on the spanwise direction, and a shift-reflections symmetry in the streamwise one (Gibson et al. 2009).

\subsection{Minimization method}

In the present work we use an minimization procedure to sample the minimal energy perturbations of the laminar state lying on the stable manifold of the edge state. Thus, we seek for the initial perturbation of the laminar state having minimal energy and lying at the minimum distance from the edge state at a given target time. To determine this distance, we first have to define a scalar product:

$$
\langle\mathbf{u}, \mathbf{v}\rangle=\int_{V} \mathbf{u} \cdot \mathbf{v} d V
$$

where $\mathbf{u}=(u, v, w)^{T}$ is the perturbation velocity vector, and $V$ indicates the whole computational domain. Then, we have to construct an objective function taking into account the initial energy of the perturbation, namely:

$$
E_{0}=\frac{1}{2}\left\langle\mathbf{u}_{\mathbf{0}}, \mathbf{u}_{0}\right\rangle
$$

where $\mathbf{u}_{0}$ is the perturbation to the laminar state at the given time $t=0$. Even if in general the edge states are time-dependent (either chaotic or periodic solutions) and are characterized by more than one unstable direction, in the chosen configuration the edge state is the lower branch exact solution of the NS equations $\mathbf{u}_{L B}$, which is steady and of codimension-one (see Nagata (1990), Schneider et al. (2008)). Thus, it is possible to define in the following way the distance of the perturbation at target time $T$ to the edge state $\mathbf{u}_{E S}=\mathbf{u}_{L B}$ (expressed as a perturbation of the laminar fixed point):

$$
d=\left\langle\left(\mathbf{u}(T)-\mathbf{u}_{E S}\right),\left(\mathbf{u}(T)-\mathbf{u}_{E S}\right)\right\rangle .
$$

The objective function thus reads:

$$
\Im=\left\langle\mathbf{u}_{0}, \mathbf{u}_{0}\right\rangle \frac{\left\langle\left(\mathbf{u}(T)-\mathbf{u}_{E S}\right),\left(\mathbf{u}(T)-\mathbf{u}_{E S}\right)\right\rangle}{\left\langle\mathbf{u}_{E S}, \mathbf{u}_{E S}\right\rangle},
$$

the first term on the numerator accounting for the initial energy, the second one for the distance from the edge state at the chosen target time, whereas the term on the denominator is a renormalization w.r.t. the edge state energy. The objective functional is minimized using a Lagrange multipliers technique, where the incompressible NS equations in a perturbed formulation have been imposed as constraint using the 
Lagrange multipliers $\left(\mathbf{u}^{\dagger}, p^{\dagger}\right)$, giving the following functional:

$$
\begin{aligned}
& \mathcal{L}=\Im-\int_{0}^{T}\left\langle p^{\dagger}, \nabla \cdot \mathbf{u}\right\rangle d t-\int_{0}^{T}\left\langle\mathbf{u}^{\dagger},\left\{\frac{\partial \mathbf{u}}{\partial t}+\mathbf{u} \cdot \nabla \mathbf{U}\right.\right. \\
& \left.\left.+\mathbf{U} \cdot \nabla \mathbf{u}+\mathbf{u} \cdot \nabla \mathbf{u}+\nabla p-\frac{\nabla^{2} \mathbf{u}}{R e}\right\}\right\rangle d t,
\end{aligned}
$$

where $\mathbf{U}(y)$ is the Couette base flow.

In order to find the minimum of the functional, its first variation with respect to all of the variables must be set to zero. In particular, zeroing the first variation of the functional with respect to the direct and adjoint variables provides the direct and the adjoint equations, as well as the following compatibility conditions:

$$
\frac{2 u^{\prime}}{E_{0}}-b=0, \frac{2 v^{\prime}}{E_{0}}-c=0, \frac{2 w^{\prime}}{E_{0}}-d=0, \text { for } t=T .
$$

Since the NS equations are non-linear, the adjoint equations are linked to the direct ones by the presence of direct variables in the advection terms; thus, the whole direct field needs to be stored at each time step, requiring a very large storage capacity. The direct and adjoint equations are parabolic in the forward and backward time direction, respectively, so that they can be solved by a coupled iterative approach. Moreover, since the output of the minimation is a perturbation at $t=0$, the gradient of $\mathcal{L}$ with respect to the initial disturbance $\mathbf{u}_{0}$ has to vanish within a reasonable number of iterations. In order to achieve convergence efficiently, a conjugate gradient algorithm is used, similar to that employed in Cherubini et al. (2011). The initial perturbation is updated in the steepest descent direction, denoted as $\nabla_{\mathbf{u}_{0}} \mathcal{L}$, namely:

$$
\begin{aligned}
& \frac{\partial \mathcal{L}}{\partial u_{0}}=-2 u_{0} \frac{\left\langle\left(u(T)-u_{E S}\right),\left(u(T)-u_{E S}\right)\right\rangle}{\left\langle u_{E S}, u_{E S}\right\rangle}+u^{\dagger}(0), \\
& \frac{\partial \mathcal{L}}{\partial v_{0}}=-2 v_{0} \frac{\left\langle\left(v(T)-v_{E S}\right),\left(v(T)-v_{E S}\right)\right\rangle}{\left\langle v_{E S}, v_{E S}\right\rangle}+v^{\dagger}(0), \\
& \frac{\partial \mathcal{L}}{\partial w_{0}}=-2 w_{0} \frac{\left\langle\left(v(T)-v_{E S}\right),\left(w(T)-w_{E S}\right)\right\rangle}{\left\langle w_{E S}, w_{E S}\right\rangle}+w^{\dagger}(0),
\end{aligned}
$$

with an adjustable step length $\alpha$, so that $\mathbf{u}_{0}^{(n+1)}=\mathbf{u}_{0}^{n}-\alpha^{n} \nabla_{\mathbf{u}_{0}} \mathcal{L}^{n}$. After the first iteration in the steepest descent direction the successive steps are taken along a conjugate direction, $\Lambda \mathbf{u}_{0}$, which is computed on the basis of the gradient at two consecutive iterations according to $\Lambda \mathbf{u}_{0}^{(n+1)}=\nabla_{\mathbf{u}_{0}} \mathcal{L}^{(n+1)}+\beta^{(n+1)} \Lambda \mathbf{u}_{0}^{n}$. In the present work the value of the parameter $\beta^{(n+1)}$ is computed by means of the Polak-Ribière formula (Polak \& Ribière 1969). Periodically, this value should be reset to zero in order to avoid conjugacy loss (which corresponds to updating the solution in the steepest descent direction). The step length $\alpha$ has been chosen small in order to ensure convergence to the edge state at the target time, as described below. Moreover, in order to keep the perturbation on the laminar-turbulent boundary, a threshold value for the distance to the edge state has been chosen, namely, $10^{-5}$. When such a threshold is exceeded, the last iteration is repeated multiplying the gradient term by a factor 0.5. Essentially, to keep the perturbation at such a small distance from the edge state, the initial perturbation has been updated in 
the conjugate gradient direction by a very small amount at each iteration, so that nearly 40000 iterations are needed to reach convergence at a given target time, making the problem very computationally expensive. When convergence is achieved, the minimal initial perturbation $\mathbf{u}_{T}^{\min }$ is on the stable manifold of the edge state, reaching it at time $T$ within a distance of at most $10^{-5}$. Varying the time horizon, we are able to sample the stable manifold by characterizing the local minima of disturbance kinetic energy.

The optimization procedure for a chosen target time $T$ can be summarized as follows:

(i) The initial guess for the initial condition, $\mathbf{u}_{0}$, is given by the edge state itself.

(ii) The direct problem is integrated up to $t=T$.

(iii) The adjoint variables, $\left(\mathbf{u}^{\dagger}, p^{\dagger}\right)$ are provided by the compatibility conditions $(7)$.

(iv) The adjoint problem is integrated backward in time from $t=T$ to $t=0$.

(v) At $t=0$, the initial direct variables are updated in the direction of the conjugate gradient with step length $\alpha$ and $\beta$ computed according to the Polak-Ribière formula ( $\beta=0$ is imposed at the first iteration).

(vi) The objective function $E(T)$ and the distance $d$ from the edge are evaluated:

(a) if the gradient $\nabla_{\mathbf{u}_{0}} \mathcal{L}$ is smaller than a chosen threshold, the loop is stopped, otherwise the procedure is continued from step $(i i)$;

(b) if the distance $d$ is larger than a chosen threshold, $d>10^{-5}$, the value of $\alpha$ is halved, the value of $\beta$ is set to zero, and the procedure is continued from step (ii) using the gradient computed at the previous iteration.

\section{Results}

The minimal energy perturbations on the stable manifold of the LB solution for $R e=400$ are computed for 6 target times, $T=5,20,40,60,80,100$. The top-left frame of Figure 2 shows the value of the minimal energy versus the target time. As shown by the solid blue line in the inset, the minimal energy tends to an asymptotic value for large target times. Plotting the curve on a double logarithmic scale, a linear slope is recovered (black dotted line). Thus, it appears that, for small enough target times $(5<T<100), E_{\text {min }}$ varies with $T$ following a power law, whose best fit is given by $E_{\text {min }} \propto T^{-\frac{7}{4}}$. We have verified that for a larger target time, $T=200$, the initial minimum energy computed by using the method in Pringle \& Kerswell (2010) follows the same power law. However, this law cannot be valid for $T \rightarrow \infty$, since the flow is linearly stable. The shape of the minimal perturbations is given in figure 2 , in the subframes from (b) to (e) for $T=5,20,60,100$, respectively. For the smallest target time, $T=5$, the minimal perturbation obviously preserves the symmetries that characterize the LB state. For $T=20$, the shift-reflection symmetry is broken, but the perturbation is still invariant with respect to a finite translation in the spanwise direction. Moreover, the streamwise perturbation velocity (blue and pink for negative and positive values) begins 

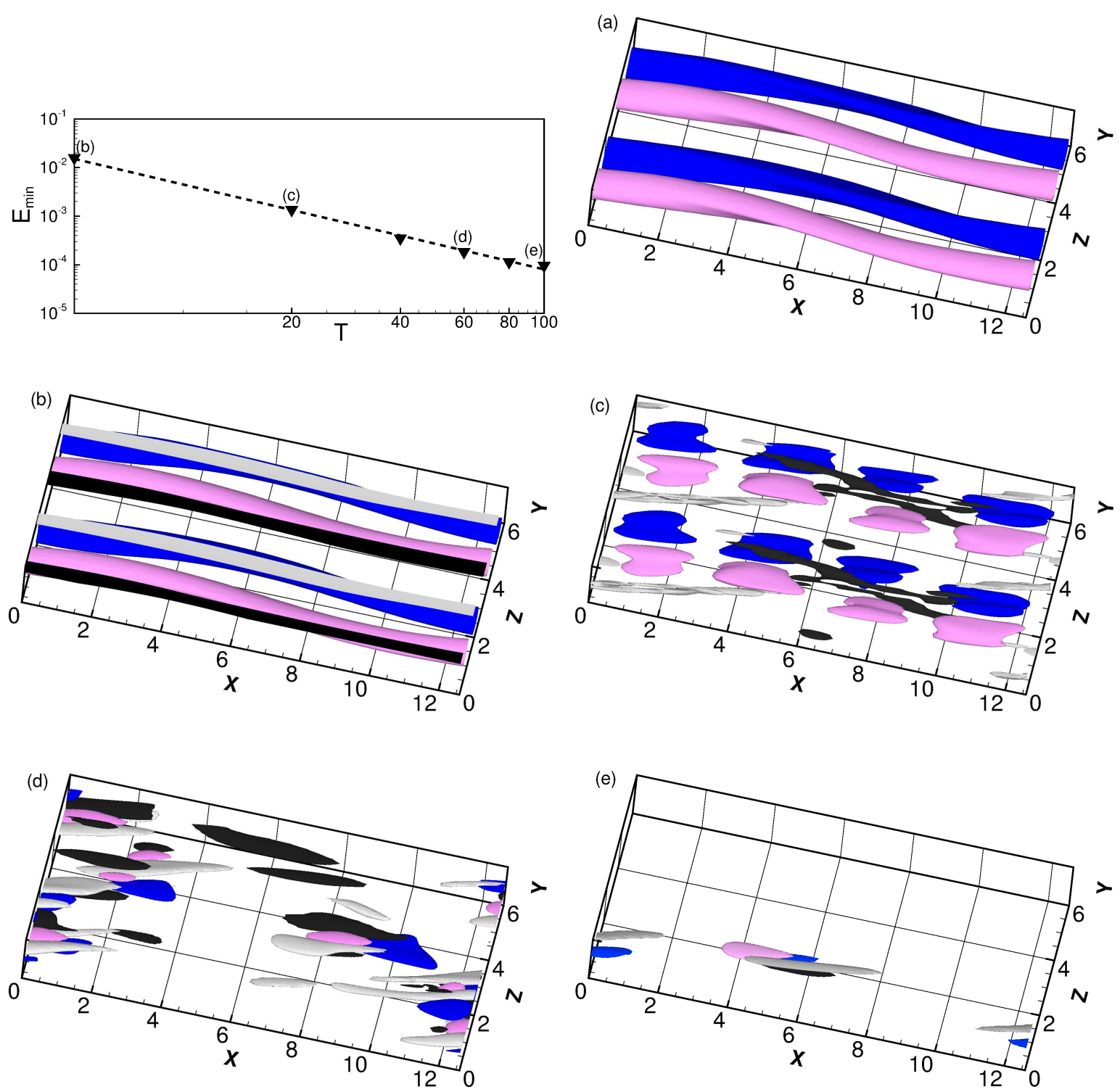

Figure 2. Minimal perturbation energy versus target time in a double logarithm scale: the triangles are the results of the minimisation procedure, the dashed line represents the most accurate power fit, whereas the asymptotic value of $E_{0}$ is shown in the inset. The structure of the edge state (a) and of the minimal perturbations obtained for (b) $T=5$, (c) $T=20$, (d) $T=60$, and (e) $T=100$ is provided. The white and black surfaces represent the positive and negative streamwise component of the vorticity perturbation $\left(\omega_{x}= \pm 1.7,1.7,0.9,0.4,0.37\right.$ from (a) to (e)); pink and blue represent the positive and negative streamwise velocity component $(u= \pm 0.3,0.3,0.08,0.045,0.035$ from (a) to (e)). 
to be more localized in the streamwise direction, showing alternated patches instead of streaky structures. For $T \geq 40$, also the spanwise translation symmetry is broken, and the perturbation begins to localize also in the spanwise direction. In particular, a repeated basic structure can be identified, which is characterized by scattered patches of streamwise velocity with inclined streamwise vortices (black and white) on their flanks. Further increasing the target time, a stronger localization in both the streamwise and spanwise directions is observed, as shown in subframe (d) for $T=100$. However, the shape of the minimal perturbation remains characterized by a similar basic structure, which strongly recall the minimal seed of turbulent transition found in Cherubini \& De Palma (2013) for pCf, and in Cherubini et al. (2010) for a boundary-layer flow. This indicates that for large enough target times the minimal perturbations approach the minimal seed, but they largely differ from it at short times.

The dynamics of the evolution of the minimal perturbations towards the edge state can be analysed by looking at the trajectories on the energy input $(I)$ versus dissipation rate $(D)$ plane (Kawahara \& Kida 2001). All of the trajectories appear to have an origin close to the bisector (dashed line), where $\dot{E}=I-D$ is close to zero, as shown in figure 3 for $\mathbf{u}_{T=20,60,100}^{\min }$. Then, the trajectories pierce into the top half of the $I-D$ plane, strongly increasing their dissipation, before relaxing towards the edge state represented by the black circle on the bisector. As shown by the correlation values (Kerswell \& Tutty 2007) in the inset of figure 3 , the trajectories spend a considerable amount of time close to the edge $(C \approx 1)$, before escaping from it along its unstable manifold towards the top half of the $I-D$ plane, where $D>I$. Eventually, they reach the chaotic saddle, as shown by the disordered trajectory around $D \approx I \approx 2.5$. Concerning the transition time, the white symbols in figure 3 indicate time intervals of 10 units, from $t=0$ to $t=250$. One can observe that each perturbation reaches the edge state, and then chaos, at a very different time. However, the escape route from the edge is common (within a tolerance level) to all of the computed trajectories, since the unstable manifold of the edge state has codimension one.

The fact that all of the minimal energy perturbations strongly dissipate their energy before reaching the edge state can be interpreted in terms of the coherent structures produced during their evolution. It is known that the LB state is self-sustained by a process based on the mutual generation of streaks and vortices (Wang et al. 2007). Thus, the evolution of the energy associated with streaky and vortical structures may provide some clues on the establishment of such a self-sustained process. Figure 4 provides the evolution of the crossflow energy:

$$
E_{c f}=\frac{1}{V} \int_{V}\left(v^{2}+w^{2}\right) d V
$$

associated with the vortices, versus the longitudinal energy:

$$
E_{l}=\frac{1}{V} \int_{V} u^{2} d V
$$

associated with the streaks. At $t=0, E_{c f}$ is rather low, then it strongly increases in a very small time and finally decreases to values comparable to the initial ones, when 


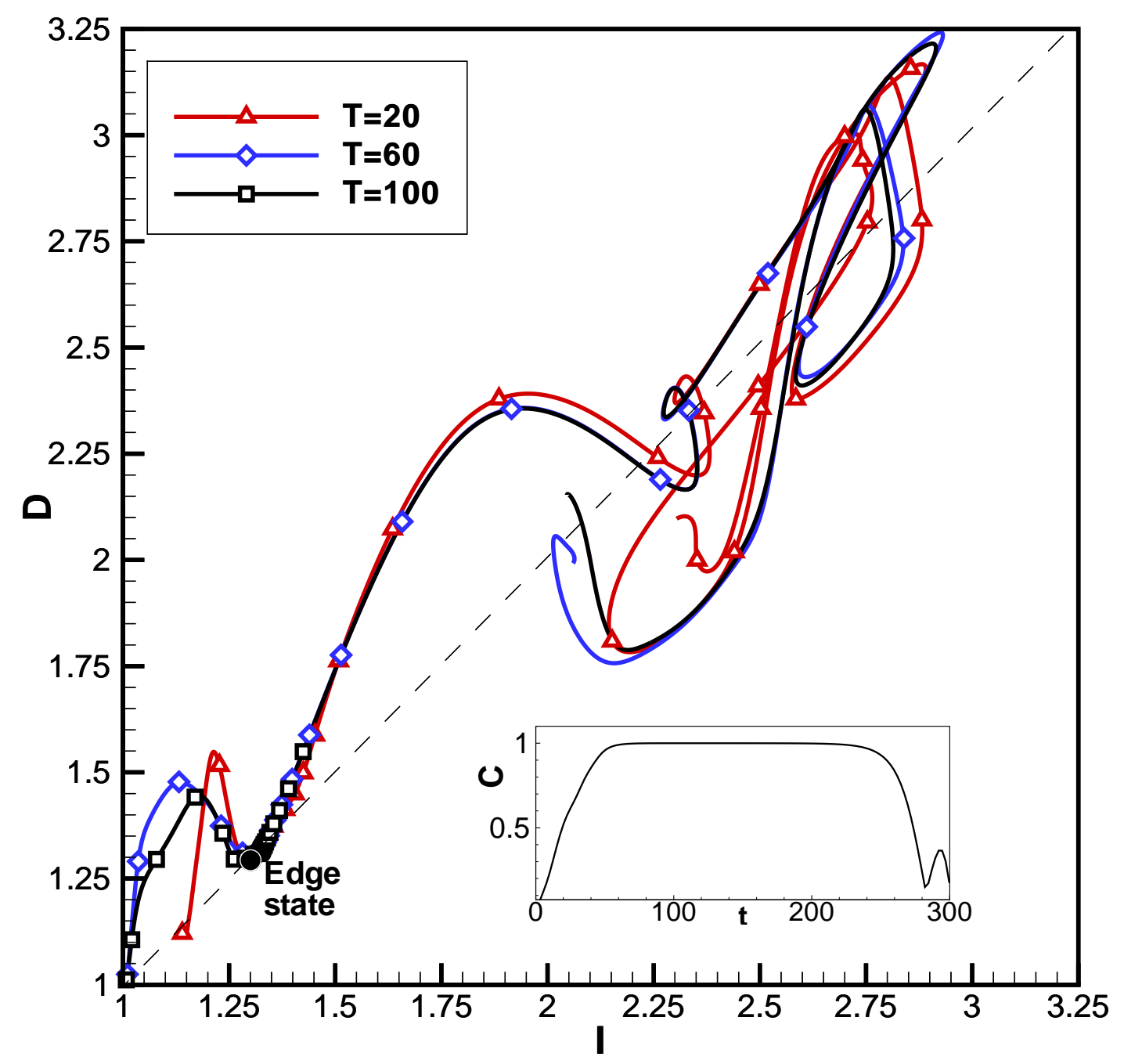

Figure 3. Trajectories of the minimal perturbations $\mathbf{u}_{T=20,60,100}^{\min }$ in the dissipationrate versus energy-input plane. The white triangles, squares and diamonds indicate time intervals of 10 units, from $t=0$ to $t=250$, for the minimal perturbations determined for $T=20,60,100$, respectively. The inset shows the correlation with the edge state versus time corresponding to $\mathbf{u}_{T=100}^{\min }$.

relaxing on the edge state (see the plateau for $E_{l} \approx 6$ ). Moreover, $E_{c f}$ peaks almost at the same time of the dissipation rate, meaning that the strengthening of the vortices induces the increase of the energy dissipation. On the other hand, the longitudinal energy keeps increasing from $t=0$ to $T$, since streaks are continuously fed by the vortices due to the lift-up effect. Thus, it appears that, to keep the initial energy to a minimum, a perturbation of the laminar state must be initially characterized by low values of $E_{c f}$ and $E_{l}$, but it should be able to strongly increase its crossflow energy in very small times, in order to generate longitudinal energy in the form of bent streaks. The structures created by the minimal perturbations can be observed in the subframe (a) of figure 4 for $\mathbf{u}_{T=20}^{\min }$ at $t=10$, and in subframes (b) and (c) for $\mathbf{u}_{T=100}^{\min }$ at $t=10$ and 30, respectively. Concerning $\mathbf{u}_{T=20}^{\min }$, it appears that this perturbation maintains in time its 


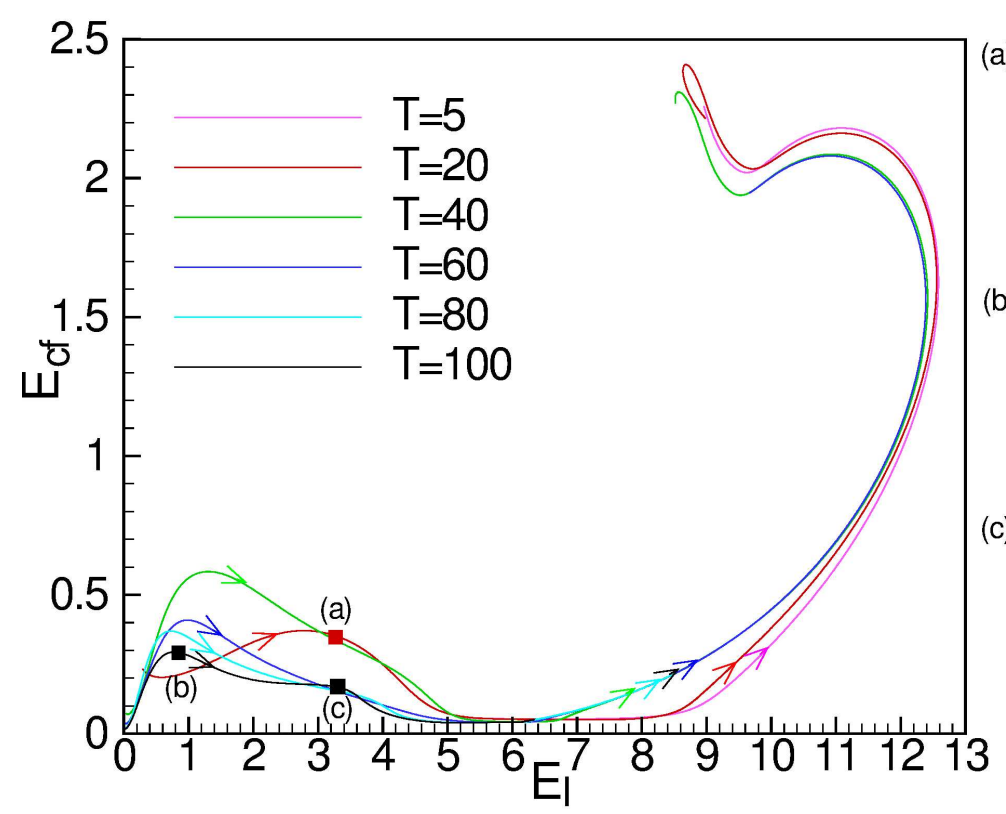

(a)

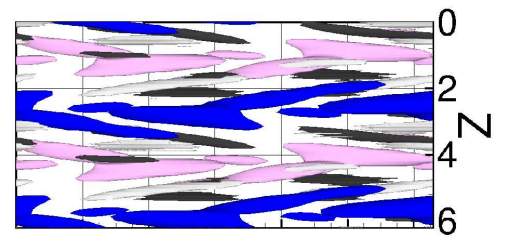

(b)

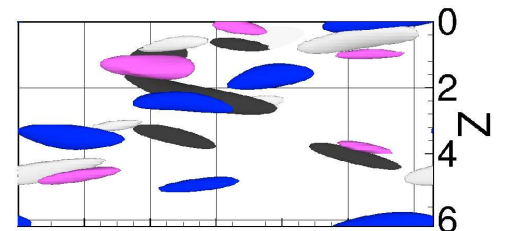

(c)

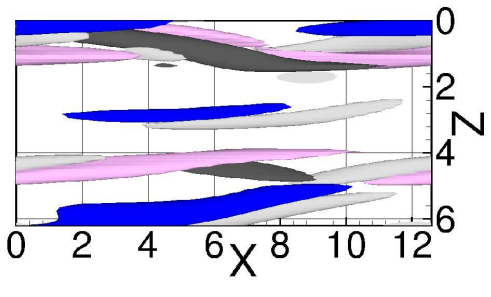

Figure 4. Longitudinal energy $\left(E_{l}\right)$ versus crossflow energy $\left(E_{c f}\right)$ for the minimal perturbations computed for $T=5,20,40,60,80,100$. The squares indicate the times at which the snapshots on the right have been extracted, namely: (a) $t=10$, for $\mathbf{u}_{T=20}^{\min }$, and (b) $t=10,(\mathrm{c}) t=30$, for $\mathbf{u}_{T=100}^{\min }$.

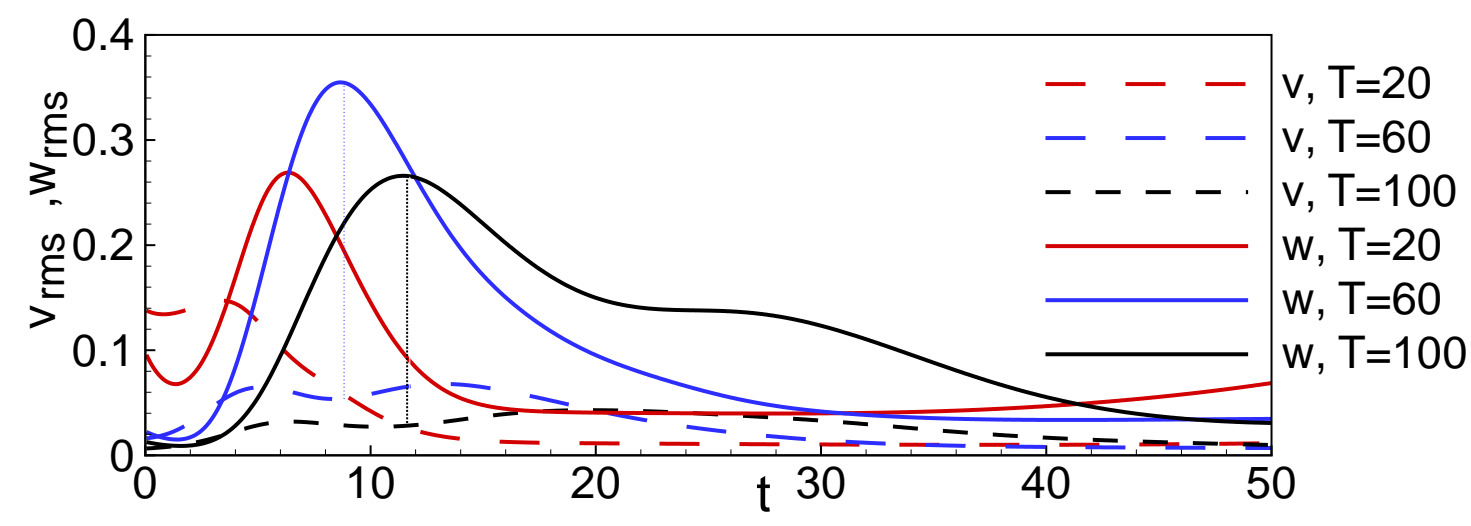

Figure 5. Evolution of $v_{r m s}$ and $w_{r m s}$ versus time for $\mathbf{u}_{T=20,40,100}^{\min }$. The dotted lines indicate that when $w_{r m s}$ peaks, $v_{r m s}$ shows a local minimum.

initial spanwise translation symmetry, and reaches at $t=10$ the shift-reflect symmetry which characterizes the edge. On the other hand, $\mathbf{u}_{T=100}^{\min }$ follows a very different route towards the edge state, since no symmetry is reached until the perturbation gets very close to the edge (see subframe $(\mathrm{c})$ for $t=30$ ). It is worth noticing that the route followed by the minimal perturbations which preserves at least one of the symmetries of the edge state (namely, $\mathbf{u}_{T=5,20}^{\min }$ ) is different from the path followed by the other ones. Figure 4 shows that the trajectories of $\mathbf{u}_{T=5,20}^{\min }$ in the $E_{c f}-E_{l}$ plane are very close to each other; also, the trajectories of $\mathbf{u}_{T=40,60,80,100}^{\text {min }}$ have a very similar shape. As target time decreases, their $E_{c f}$ peak increases and is reached at sligthly larger values of $E_{l}$. 
For $T=20$, the $E_{c f}$ peak has a lower value and is reached for much larger values of $E_{l}$. Such a different behaviour can be better observed in figure 5 where the rms values of the wall-normal and spanwise components of velocity are plotted; they account for the streamwise vortices and the bending of the streaks, respectively. For $T \geq 40$ the $v_{r m s}$ curve has a double peak, whereas $w_{r m s}$ has only one peak which is placed in correspondence with the $v_{r m s}$ local minimum. Moreover, the initial values of $v_{r m s}$ and $w_{r m s}$ are comparable. On the other hand, for $T<40, v_{r m s}$ has an initial value larger than $w_{r m s}$ and shows only one peak. Finally, the maximum value of $w_{r m s}$ does not correspond to a local minimum of $v_{r m s}$. This confirms that minimal perturbations achieving the edge in a very short time follow a very different route with respect to the ones which have a larger time to reach it. Minimal perturbations for $T<40$ have to maintain at least one of the edge state symmetries to be able to reach it in a very short time. Therefore, they cannot be localized in space and they are characterized by larger values of the velocity components. In contrast, minimal perturbations for $T \geq 40$ can be localized in space and characterized by much lower values of the velocity components, relying on energy growth mechanisms to reach the edge of chaos (Pringle \& Kerswell 2010, Cherubini et al. 2010). Although the route to turbulence of such minimal perturbations is very different, we have found that their inital energy follows a power law with respect to the target time. This allows to establish a threshold for the minimal initial energy needed to reach the edge of chaos at a certain (short) given time.

\section{Summary}

In this work we have provided aninvestigation of the structure of the laminar-turbulent boundary for the case of the plane Couette flow, using the time as parameter. These results could be used to develop a control strategy based on the stabilization of the flow on the edge state with very low-dimension unstable manifold (Wang et al. 2007). The knowledge of the shape and energy of the minimal perturbation on the stable manifold of the LB state could be employed to design a suitable control strategy, inspired by the feedback approach for chaotic systems of Pyragas (Pyragas 1992), to stabilize the low-dissipation LB solution using MEMS technology for sensors ad actuators. Future works will aim at extending the present technique to time-dependent edge states, for instance, using a Poincaré map formulation in the case of periodic orbits.

\section{Acknowledgments}

Some computations have been performed on the Power 6 of the IDRIS, France. The authors would like to thank A. Bottaro, D. Henningson, and O. Semeraro for useful discussions about this work. 


\section{References}

Cherubini S \& De Palma P 2013 J. Fluid Mech. 716, 251-279.

Cherubini S, De Palma P, Robinet J \& Bottaro A 2010 Phys. Rev. E 82, 066302.

Cherubini S, De Palma P, Robinet J C \& Bottaro A 2011 J. Fluid Mech. 689, 221-253.

Clever R M \& Busse F H 1992 J. Fluid Mech. 234, 511-527.

Cossu C 2005 C. R. Mcanique 333, 331-336.

Duguet Y, Brandt L \& Larsson B R J 2010 Phys. Rev. E 82, 026316.

Duguet Y, Monokrousos A, Brandt L \& Henningson D S 2013 Phys. Fluids 25, 084103.

Eckhardt B, Schneider T M, Hof B \& Westerweel J 2007 Annu. Rev. Fluid Mech. 39, 447-468.

Gibson J F, Halcrow J \& Cvitanović P 2009 J. Fluid Mech. 638, 243.

Hof B, van Doorne C, Westerweel J, Nieuwstadt F, Faisst H, Eckhardt B, Wedin H, Kerswell R \& Waleffe F 2004 Science 305, 1594-1598.

Kawahara G 2005 Phys. Fluids 17, 041702.

Kawahara G \& Kida S 2001 J. Fluid Mech. 449, 291-300.

Kerswell R \& Tutty R O 2007 J. Fluid Mech. 584, 69-101.

Monokrousos A, Bottaro A, Brandt L, Di Vita A \& Henningson D S 2011 Phys. Rev. Lett. 106, 134502.

Nagata M 1990 J. Fluid Mech. 217, 519-527.

Polak E \& Ribière G 1969 Rev. Francaise Informat Recherche Operationnelle 16, 35-43.

Pringle C C T \& Kerswell R 2007 Phys. Rev. Lett. 99, 074502.

Pringle C C T \& Kerswell R 2010 Phys. Rev. Lett. 105, 154502.

Pringle C C T, Willis A P \& Kerswell R 2012 J. Fluid Mech. 702, 415-443.

Pyragas K 1992 Phys. Lett. A 170, 421-428.

Rabin S M E, Caulfield C P \& Kerswell R 2012 J. Fluid Mech. 712, 244-272.

Reddy S C, Schmid P J, Baggett J S \& Henningson D S 1998 J. Fluid Mech. 365, 269-303.

Reynolds O 1883 Philos. Trans. R. Soc. London Ser. A 174, 935.

Schmid P \& Henningson D 2001 Stability and transition in shear fows Springer, New York.

Schneider T M, De Lillo F, Buehrle J, Eckhardt B, Dörnemann T, Dörnemann K \& Freisleben B 2010 Phys. Rev. E 81, 015301.

Schneider T M, Gibson J F, Lagha M, De Lillo F \& Eckhardt B 2008 Phys. Rev. E 78, 037301.

Skufca J D, Yorke J \& Eckhardt B 2006 Phys. Rev. Lett. 96, 174101.

Verzicco R \& Orlandi P 1996 J. Comp. Phys. 123(2), 402-414.

Viswanath D \& Cvitanović P 2009 J. Fluid Mech. 627, 215.

Waleffe F 1997 Phys. Fluids 9, 883-901.

Waleffe F 2003 Phys. Fluids 15, 1517-1534.

Wang J, Gibson J F \& Waleffe F 2007 Phys. Rev. Lett. 98, 204501. 